\title{
Velocidad cortical y campo de esfuerzos en el Archipiélago Balear utilizando series temporales GPS de la red XGAIB (2010-2013)
}

\author{
Alberto SÁnCHEZ-AlzOlA ${ }^{1}$, CARlos SÁNCHEZ ${ }^{2}$, JORdi GiMÉnEZ ${ }^{3}$, PEDRO \\ AlFARO $^{4}$, Bernadí GElABERT ${ }^{5}$, MARÍA J. BorQUE ${ }^{6,7}$, ANTONIO J. GIL $^{6,7}$ \\ ${ }^{1}$ Dpto. de Estadística e I.O. Universidad de Cádiz. alberto.sanchez@uca.es \\ ${ }^{2}$ Serveis d'Informació Teritorial de les Illes Balears (SITIBSA) \\ ${ }^{3}$ Servei d'Estudis i Planificació, D.G. Recursos Hidrics \\ ${ }^{4}$ Dpto. de Ciencias de la Tierra y del Medio Ambiente, Universidad de Alicante \\ ${ }^{5}$ Dpto. de Ciencias de la Terra. Universitat de les Illes Balears \\ ${ }^{6}$ Dpto. de Ingeniería Cartográfica, Geodésica y Fotogrametría, Universidad de Jaén \\ ${ }^{7}$ Centro de Estudios Avanzados en Ciencias de la Tierra (CEACTierra), Universidad de Jaén
}

Recibido: $31 / 07 / 2014$

Aceptado: 14/10/2014

\section{Resumen}

En este artículo se presenta la velocidad cortical y el campo de esfuerzos en la zona del archipiélago Balear en base a observaciones GPS continuas de la red XGAIB en el período 2010-2013. La red XGAIB está compuesta por nueve estaciones GPS permanentes distribuidas de manera homogénea por el archipiélago y se encuentra activa desde el año 2010. En esta contribución se describe la red XGAIB y el proceso de cálculo con las estaciones GPS continuas (CGPS) realizado, presentando las velocidades absolutas y relativas para todas las estaciones. Además se ha estimado el campo de esfuerzos para obtener un modelo de deformación cortical realista del archipiélago. Los resultados muestran una variación gradual de los esfuerzos sobre las Islas Baleares, desde la extensión WNWESE presente en el suroeste (Islas Pitiusas) hasta la compresión NW-SE del nordeste (Isla de Menorca). Estos resultados constituyen un avance en el conocimiento de la tectónica en la región más occidental del Mediterráneo.

Palabras clave: CGPS, posicionamiento puntual de precisión, deformación cortical, series temporales, Islas Baleares.

\section{Crustal velocity and strain rate fields in the Balearic Islands based on GPS time series from the XGAIB network (2010-2013)}

\begin{abstract}
In this paper, a first estimation of the crustal velocity and strain rate fields in the Balearic Islands is presented. This study is based on continuous GPS observations from the XGAIB network spanning the period 2010-2013. The XGAIB network consists of nine permanent, widely distributed stations that have operated continuously since 2010 . We describe the network, the continuous GPS (CGPS) data processing and present our principle results in terms of absolute and residual velocities of all of the sites. In addition, strain tensors were estimated from the velocity field to obtain the first realistic crustal deformation model of the archipelago. The strains exhibit gradual variation across the Balearic Islands, from WNW-ESE extension in the southwest (Pitiusas Islands) to NW-SE compression in the northeast (Menorca Island). These results constitute an advance in the knowledge of the tectonics of the western Mediterranean region.
\end{abstract}


Keywords: CGPS, precise point positioning, crustal deformation, time series, Balearic Islands.

Sumario: Introducción. 1. Marco geodinámico. 2. Datos CGPS. 3. Metodología. 4. Resultados. 5. Discusión. 6. Conclusiones. Agradecimientos. Referencias.

\section{Referencia normalizada}

Sánchez-Alzola, A., Sánchez, C., Giménez, J., Alfaro, P., Gelabert, B., Borque, M. J., Gil, A. J. (2014). Velocidad cortical y campo de esfuerzos en el Archipiélago Balear utilizando series temporales GPS de la red XGAIB (2010-2013). Física de la Tierra, Vol. 26, 47-63.

\section{Introducción}

La parte más occidental del Mediterráneo es una región de gran interés para la investigación ya que se trata de un área de compleja geodinámica donde confluyen las placas Euroasiática y Africana. En los últimos años se han planteado diversos modelos para conseguir estudiar la geología, geofísica y geodinámica de esta región (Argus y Gordon., 1991; Dewey et al., 1989; DeMets et al., 1994; Calais et al., 2003). Más recientemente, los nuevos trabajos han incluido datos geodésicos que han permitido mejorar la precisión de estos modelos (McClusky et al., 2003; Nocquet y Calais, 2003; Stich et al., 2006; Fadil et al., 2006; Serpelloni et al., 2007; Fernandes et al., 2007; Tahayt et al., 2008; Perez-Peña et al., 2010; Vernant et al., 2010; Palano et al., 2011; Koulali et al., 2011). En esta zona el área de confluencia presenta una velocidad de convergencia de entre 4 a $6 \mathrm{~mm} / \mathrm{año}$, mostrando un contacto difuso en la zona próxima a la Península Ibérica, desde $l o s ~ 12^{\circ} \mathrm{W}$ a $1^{\circ} \mathrm{W}$ de longitud. En la parte más oriental, el contacto se concentra en una estrecha área de la zona central de la región del Maghrib (Argelia) al sur de las Islas Baleares (desde $\operatorname{los} 1^{\circ} \mathrm{E}$ a $7^{\circ} \mathrm{E}$ de longitud). Al norte de esta área la zona del archipiélago Balear parece acomodar únicamente una pequeña parte de esta convergencia, la cual se caracteriza por una baja sismicidad. Esta actividad reducida es la principal razón de la presencia de pocos estudios sobre la tectónica de la zona (Silva et al., 1997, 1999, 2000, 2001, 2004, 2005; Giménez y Gelabert, 2002; Giménez, 2003; Gelabert et al., 2005) aunque otros estudios describen una actividad de tipo Plio-Cuaternario en el conjunto del promontorio del archipiélago (Roca, 1996; Acosta et al., 2001, 2002, 2004; Maillard y Mauffret, 2013).

Respecto a la información geodésica disponible, ésta se encuentra limitada a estudios GPS regionales en la zona occidental del Mediterráneo, enfocándose preferentemente en el límite de placas Euroasiática-Nubia en las proximidades del Estrecho de Gibraltar (Fadil et al., 2006; Serpelloni et al., 2007; Perez-Peña et al, 2010; Vernant et al., 2010; Palano et al., 2011; Koulali et al., 2011). De acuerdo con Serpelloni et al. (2007), el Tell Argelino se acomoda entre 2.7 a 3.9 mm/año confirmando que el acortamiento presente tiene unas magnitudes de entre 1.6 y 2.7 $\pm 0.6 \mathrm{~mm} / \mathrm{año}$. Este proceso está activo entre el norte de África y la Península Ibérica a través de la cuenca Argelo-Balear. En función de sus resultados se concluye que la convergencia no está completamente absorbida por la sísmica 
presente en el norte de África y que se transfiere y acomoda en áreas situadas más al norte.

Desde el año 2010 el Archipiélago Balear cuenta con una red GNNS activa (XGAIB) para el posicionamiento en tiempo real y la observación de datos continuos GNSS. El objetivo de esta red es dar soporte a los trabajos geodésicos, cartográficos y topográficos en la región. En este artículo se presenta un primer estudio con el modelado de la deformación actual en el archipiélago, así como el establecimiento de un campo de velocidades preliminar en esta área. La metodología utilizada se fundamenta en el uso de series temporales de posiciones CGPS y en la técnica de procesado de posicionamiento puntual de precisión (PPP). Los resultados obtenidos fueron integrados con información geológica para mejorar de este modo la comprensión de la tectónica en esta región occidental del Mediterráneo.

\section{Marco geodinámico}

Las Islas Baleares están formadas por la parte emergida del promontorio Balear, el cual se extiende en dirección SW-NE desde Ibiza hasta Menorca (Fig. 1). Estas islas han sido afectadas por al menos dos fases tectónicas desde el final del Oligoceno hasta la actualidad: (1) Desde el Mioceno Medio al Oligoceno se presentó un acortamiento NW-SE, el cual produjo una serie de estructuras compresivas en dirección NE-SE que afloran en la mayoría de las islas, (2) Desde finales del Mioceno hasta la actualidad se presentó un proceso extensivo, el cual formó un conjunto de cuencas extensionales con el desmembramiento del promontorio Balear en dos bloques separados por el Canal de Mallorca (Fig. 1).

Las evidencias de actividad tectónica reciente son muy escasas en todo el promontorio. En base al grosor del sedimento acumulado en la cuenca de Inca (Mallorca) durante el Neógeno, el promedio de desplazamiento vertical de estas estructuras desde el Mioceno hasta el presente es de $0.1 \mathrm{~mm} / \mathrm{año}$ (Giménez y Gelabert, 2002). La evidencia de actividad tectónica reciente en Mallorca se concentra en la parte central de la isla y se relaciona con estructuras NE-SW que interactúan con estructuras menores en dirección NW-SE. De acuerdo con Sàbat et al. (2011), la extensión del final del Mioceno es perpendicular a las fallas extensionales NNE-SSW. La alineación de las cuencas y horsts localizados entre la Sierra de Tramuntana y la zona central de Mallorca se interpreta con el movimiento sinistrorso de las fallas principales NE-SE de la isla (Giménez y Gelabert, 2002; Giménez, 2003).

En Menorca, la extensión del Neógeno no es tan evidente como ocurre en la Isla de Mallorca. De acuerdo con Gelabert et al. (2005), la parte más meridional de la isla está representada prácticamente por completo por calcarenitas del Mioceno superior y está dominada por un gran anticlinal NNE-SSW cuya estructura se relaciona con la inversión tectónica de una falla normal NNE-SSW en el Neógeno. 
En las Islas Pitiusas las estructuras post-Alpinas son incluso menos evidentes debido a la baja actividad tectónica que presentan. En la isla de Ibiza, las estructuras compresivas están fracturadas por fallas NNW-SSE mientras que en Formentera, donde las rocas más antiguas datan del final del Mioceno, no se observan evidencias de actividad reciente. En este caso la morfología de la isla está controlada por fracturas con dirección NE-SW y NW-SE. Varias investigaciones describen además la presencia de fallas de tipo normal y de salto en dirección afectando a materiales Plio-Cuaternarios de la zona litoral (Roca, 1996; Acosta et al., 2001, 2002 y 2004; Maillard y Mauffret, 2013).

Gran parte de la actividad sísmica registrada se localiza en la parte central del promontorio Balear (Mallorca y Canal de Mallorca), caracterizada por una magnitud baja o moderada asociada generalmente a fallas NE-SW. En Mallorca la sismicidad histórica registrada ha estado concentrada en el interior de la isla asociada a fallas NE-SW activas en el Neógeno (Fig. 1). La sismicidad de Menorca, por su parte, está asociada a estructuras ENE-WSW que separan las regiones del Migjorn y Tramuntana. Respecto a las Islas Pitiusas, no se han registrado terremotos significativos en el área, siendo la actividad más cercana la que se localiza más al nordeste en los canales de Mallorca e Ibiza (Fig. 1).

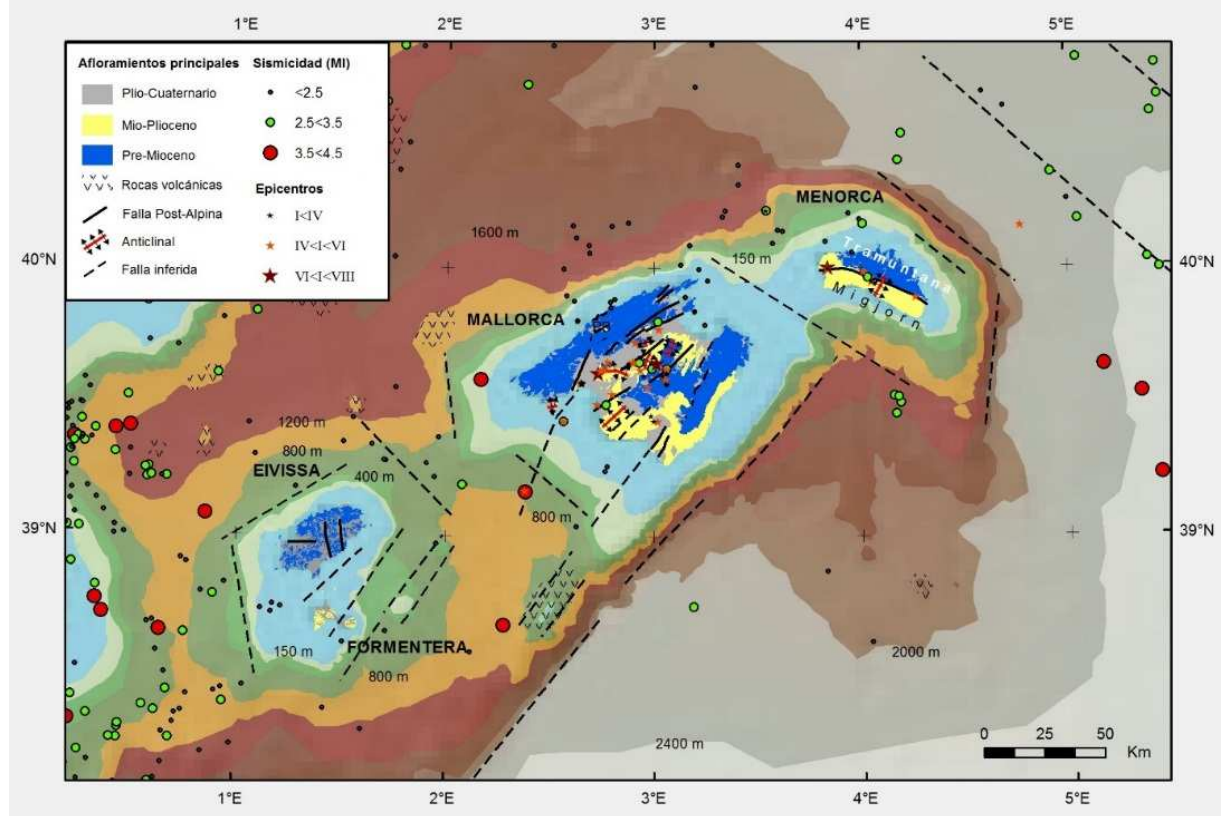

Fig. 1. Estructuras post-alpinas en el Archipiélago Balear. Quedan registrados los seísmos en el archipiélago en base al catálogo del Instituto Geográfico Nacional desde los $0.5^{\circ} \mathrm{W}$ hasta $\operatorname{los} 6^{\circ} \mathrm{E}$ de longitud, y los datos provenientes del European Mediterranean Seismological Centre (EMSC) entre los $6^{\circ} \mathrm{E}$ y los $8^{\circ} \mathrm{E}$ de longitud. Se incluyen también los epicentros sentidos (escala MSK). 
Desafortunadamente no se dispone de mecanismos focales para los seísmos de las islas. El mecanismo focal más cercano fue estimado para un terremoto localizado aproximadamente a $100 \mathrm{~km}$ al norte de la Isla de Menorca, consistente con una estructura vertical NE-SW o NW-SE con movimiento puro de salto en dirección (Braunmiller et al., 2000). Los mecanismos focales de los terremotos localizados entre las Islas Baleares y la costa más oriental de la Península Ibérica en la Provincia de Alicante, y aquellos localizados en el sur de la cuenca Argelina, están asociados a fallas E-W. Los terremotos del área del Golfo de Valencia, por su parte, están principalmente asociados con fallas normales N-S. Un número elevado de estos mecanismos focales son consistentes con la presencia de compresión N-S y extensión ortogonal. La orientación de las estructuras presentes determina la naturaleza de estas fallas de salto en dirección con direcciones NE-SW y NW-SE y las fallas normales N-S (Fig. 1).

\section{Datos CGPS}

En los últimos años se ha producido un incremento significativo en el número de regiones y comunidades autónomas que cuentan con redes GNSS activas para el posicionamiento en tiempo real, trabajos geodésicos, cartográficos y topográficos. La comunidad autónoma de las Islas Baleares es una de las que posee una de las redes más modernas, activa desde el año 2010. La red XGAIB (Xarxa de Geodèsica Activa de les Illes Balears) es una red geodésica activa gestionada por el Serveis d'Informació Territorial de les Illes Balears SITIBSA (http://xarxagnss.caib.es). Formada por nueve estaciones GNSS permanentes, esta red está operativa desde 2010 y tiene como objetivo principal el de proveer de correcciones diferenciales RTK y de observaciones espaciales para trabajos en post-proceso.

Las estaciones XGAIB están distribuidas de manera homogénea a través de las islas del archipiélago. Existen cinco estaciones en la isla de Mallorca ("MALL" (Palma de Mallorca), "TRAM" (Serra de Tramuntana), "SINE" (Sineu), "JORD" (Colonia Sant Jordi) y "BONA" (Cala Bona)), dos en la isla de Menorca ("MENC" (Ciutadella de Menorca) y "ALOR" (Alaior)), una en la isla de Ibiza ("EIVI") y una en la isla de Formentera ("FORM"). La estación de MALL (Mallorca) está gestionada a su vez por la red permanente EUREF (EPN) desde el año 2000 y posee un receptor LEICA GRX1200GGPRO con antena choke ring LEIAT504. El resto de estaciones por su parte disponen de receptores LEICA GRX1200+GNSS y antenas choke ring LEIAR25. Esta red, que cuenta además con coordenadas en el sistema de referencia ETRS89, no ha sufrido cambios de antena desde sus inicios. La Fig. 2 muestra la distribución de las estaciones XGAIB y EUREF en las Islas Baleares.

Las estaciones se encuentran localizadas sobre formaciones geológicas de varias edades y distintas tipologías de roca. Las estaciones FORM, MENC y MALL fueron construidas sobre materiales carbonatados del Mioceno Superior, la estación 
EIVI se encuentra sobre materiales del Cuaternario, la estación TRAM en materiales del Triásico, la estación SINE sobre margas burdigalianas, las estaciones BONA y JORD sobre calcarenitas del Plioceno-Cuaternario y la estación de ALOR sobre dolomitas del Jurásico.

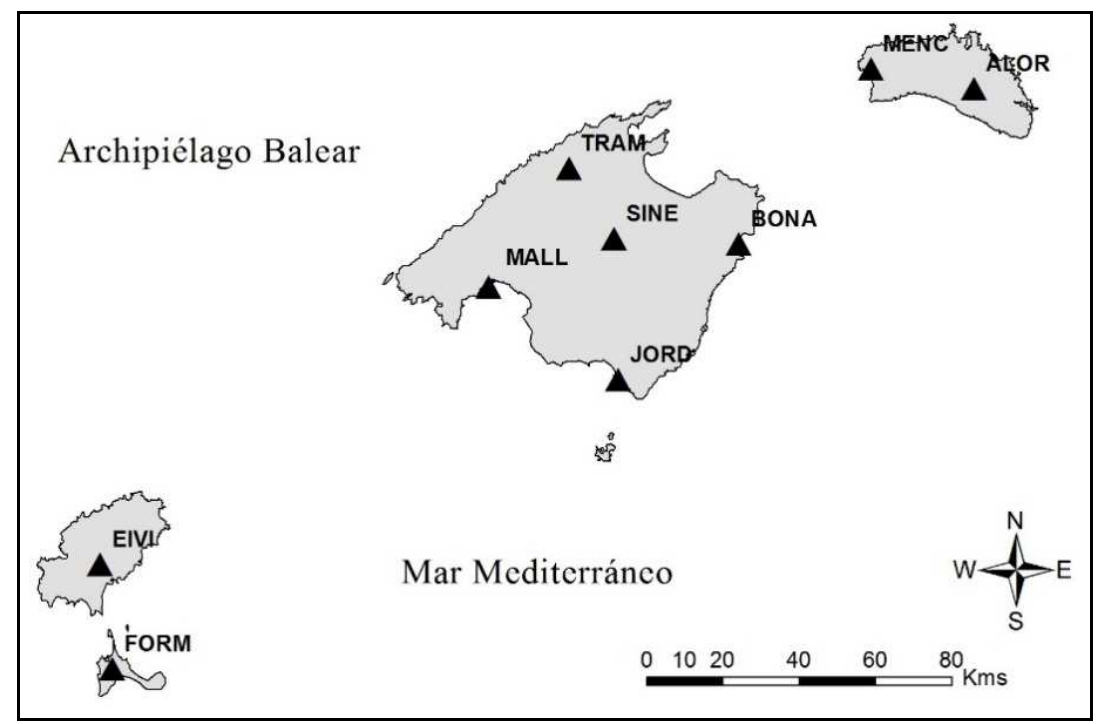

Fig. 2. Localizaciones de las nueve estaciones GNSS permanentes pertenecientes a la red XGAIB distribuidas en el Archipiélago Balear: EIVI, Ibiza; FORM, Formentera; MALL, Mallorca; TRAM, Serra de Tramuntana; SINE, Sineu; JORD, Colonia Sant Jordi; BONA, Cala Bona; MENC, Ciutadella de Menorca; ALOR, Alaior

\section{Metodología}

El método de Posicionamiento Puntual de Precisión (PPP) (Zumberge et al., 1997), que utiliza observables GPS no diferenciales, se ha convertido en una herramienta de gran utilidad para investigar procesos geofísicos a nivel sub-centimétrico (Larson et al., 2004; Smith et al., 2004; Kouba, 2005; Hreinsdóttir et al., 2006). Esta técnica ha sido utilizada en época reciente para estimar campos de velocidad en diversas regiones tectónicamente activas (Pérez et al., 2003; Teferle et al., 2007).

En este trabajo se hace uso de la versión 6.2 del software GIPSY-OASIS desarrollado por el JPL. Este programa posee un módulo básico gd2p.pl que trabaja con los datos de un único receptor aportando un posicionamiento estático puntual. Utilizando las opciones pre-establecidas en el módulo gd2p.pl según lo descrito en Bertiger et al., (2010), el software procesa los ficheros de 24 horas 30 segundos de la red XGAIB descargando todos los ficheros necesarios desde el servidor del JPL. 
Una de las ventajas más importantes de este software es el sistema de coordenadas homogéneo en el que genera las posiciones. En concreto todos los resultados obtenidos están enmarcados en el sistema IGS08, por lo que las series temporales calculadas están libres de cualquier salto u offset debido a cambios en el sistema de coordenadas. Además es posible incrementar la precisión utilizando un muestreo mayor en el fichero de reloj (hasta 5 segundos) siendo esto una ventaja adicional para series temporales de intervalo reducido o para estudios cinemáticos.

Tanto la investigación de procesos geofísicos, como el estudio sobre tectónica de placas y de deformaciones, requieren de la determinación de los parámetros de movimiento de las estaciones de manera precisa. En este trabajo ha sido posible obtener una serie temporal de datos con un intervalo de hasta tres años y medio (de marzo de 2010 hasta septiembre de 2013) con ficheros de observación a 1 segundo, que fueron posteriormente remuestreados a 30 segundos para el procesado con GIPSY-OASIS. Los datos de la estación de MALL, perteneciente a la red EUREF EPN, fueron descargados en su formato original a 30 segundos.

Respecto a las consideraciones internas del procesado, se utilizó un procedimiento estándar para todo el conjunto de datos con el módulo gd2p.pl de GIPSY-OASIS. En primer lugar se descargaron las efemérides precisas finales y los productos del polo del repositorio del JPL en el sistema de coordenadas IGS08. Para fijar las ambigüedades con PPP se utilizó la estrategia descrita en Bertiger et al. (2010) utilizando el fichero auxiliar WLPB disponible en el JPL. Además se utilizaron los ficheros de cartografiado de la troposfera GMF (Boehm et al., 2006) y los valores de contenido electrónico TEC de la ionosfera empleando un modelo de estimación basado en la Ionosfera de Referencia Internacional (IRI) (Bilitza, 2001). Para la carga oceánica se utilizó el modelo FES2004 (Lyard et al., 2006) del observatorio espacial de Onsala y para las mareas terrestres se aplicó el modelo WahrK1 (Wahr, 1985). También fueron incluidas la componente hidrostática del retardo troposférico cenital y la componente húmeda. Asimismo se aplicó el fichero de calibración de antena para corregir el centro de fase y las excentricidades, utilizando el fichero proporcionado por IGS en formato ANTEX, y se definió una máscara de elevación de $10^{\circ}$ para evitar el efecto multipath. Para el procesado se aplicó un muestreo de 300 segundos a los ficheros de observación sin ajuste de red, considerando únicamente las soluciones PPP diarias.

\section{Resultados}

Para la generación de las series temporales los resultados se transformaron a sus componentes horizontales. Los outliers que aparecieron frente a la tendencia general se eliminaron de la serie temporal utilizando un umbral específico basado en la desviación de la propia estación. Para obtener el campo de velocidades absolutas se incluyeron las series temporales en el software de análisis CATS (Williams, 2008), el cual aplica una estimación de máxima verosimilitud para 
ajustar un modelo multiparamétrico a la serie. Para establecer un mejor ajuste con el modelo, se consideraron los parámetros de periodicidad anual, semi-anual y de 13.66 días (Penna y Stewart, 2003) que dominan las series diarias (Teferle et al., 2007).

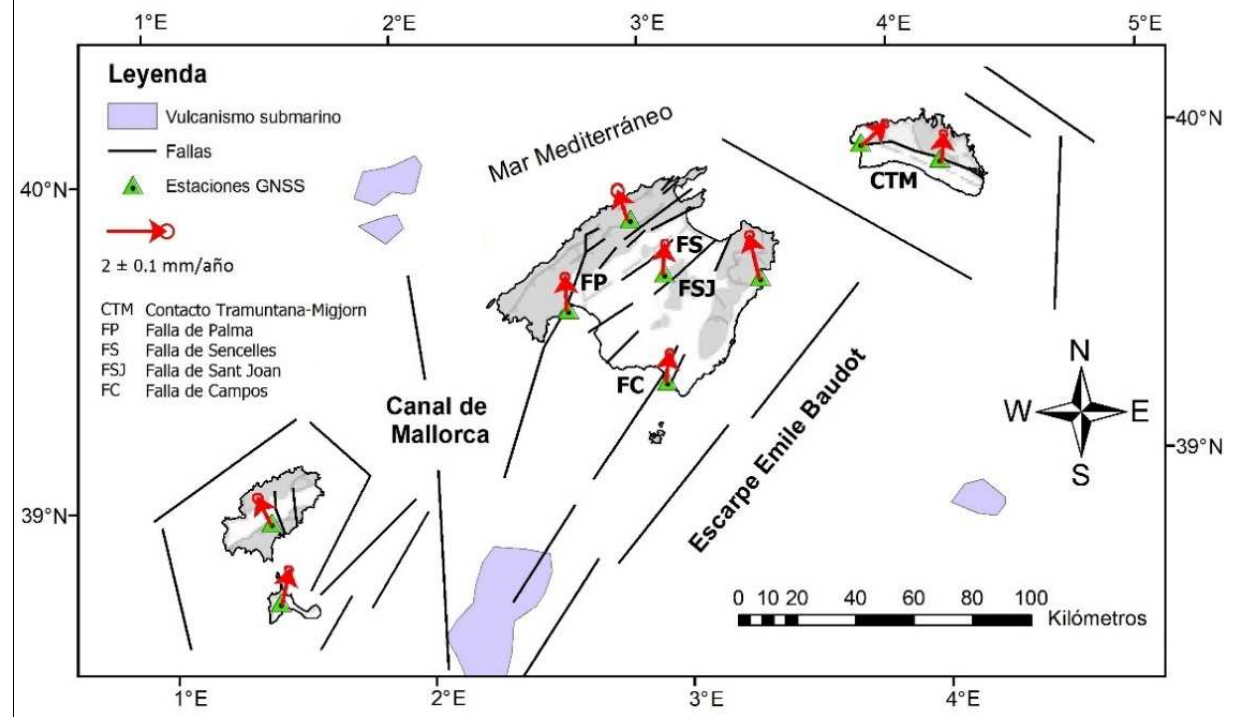

Fig. 3. Campo de velocidades residuales en mm/año respecto al modelo tectónico GEODVEL, con condiciones NNR, y la placa Euroasiática como referencia. Elipses de error definidas al $95 \%$ de confianza. Se indican también las fallas principales.

Para resaltar las diferencias entre las velocidades absolutas y el movimiento propio de la placa, se calculó el campo de velocidades residuales eliminando la componente principal de la placa Euroasiática. Para ello se utilizó el modelo tectónico GEODVEL (Argus et al., 2010) con condición NNR y la placa Euroasiática como referencia. Para este propósito se utilizó una transformación apropiada con las coordenadas geográficas de la estación como datos de entrada.

Una vez trasformadas se calculó la velocidad residual eliminando la componente principal de la placa a las velocidades absolutas obtenidas. La Tabla 1 incluye las velocidades absolutas y los errores asociados en el marco de referencia IGS08. La precisión de la estimación se calculó mediante el método de los mínimos cuadrados ponderados descrito en Koch (1999). La Fig. 3 muestra el campo de velocidades residuales respecto al modelo tectónico de referencia GEODVEL (Argus et al., 2010) con las elipses de confianza al $95 \%$.

En base al campo de velocidades absolutas previamente calculado, se han obtenido además los tensores de dilatación y extensión con los máximos esfuerzos (Voosoghi et al., 1976; Grafarend y Voosoghi, 2003; Pietrantonio y Riguzzi, 2004). Para la generación de estos resultados se utilizó el módulo de Matlab 
GRID_STRAIN (Teza et al., 2008). La Fig. 4 contiene los principales ejes de los tensores después de aplicar el cálculo de esfuerzos con el programa. La Fig.5 muestra el mapa de contorno con las zonas de predominio de acortamiento en verde y de extensión en amarillo.

Tabla. 1. Velocidades absolutas derivadas de las series temporales de posiciones con los errores asociados en el marco de referencia IGS08. Las velocidades residuales están calculadas respecto al modelo tectónico GEODVEL.

\begin{tabular}{l|cc|ccccccccc}
\hline & \multicolumn{2}{c}{$\begin{array}{c}\text { Coordenadas } \\
\text { Geográficas }\end{array}$} & \multicolumn{3}{c}{$\begin{array}{c}\text { Velocidades absolutas } \\
\text { (mm/año) }\end{array}$} & \multicolumn{3}{c}{$\begin{array}{c}\text { GEODVEL } \\
\text { (mm/año) }\end{array}$} & \multicolumn{2}{c}{$\begin{array}{c}\text { V. Residuales } \\
\text { (mm/año) }\end{array}$} \\
\hline & $\begin{array}{c}\text { Lat. } \\
(\mathrm{N})\end{array}$ & $\begin{array}{c}\text { Long } \\
\left({ }^{\circ} \mathrm{E}\right)\end{array}$ & $\mathrm{VE}$ & $\sigma \mathrm{E}$ & $\mathrm{VN}$ & $\sigma \mathrm{N}$ & $\mathrm{VE}$ & $\mathrm{VN}$ & $\mathrm{VE}$ & VN \\
\hline ALOR & 39.9344 & 4.14015 & 20.63 & \pm 0.05 & 16.26 & \pm 0.04 & 20.50 & 15.31 & 0.13 & 0.95 \\
BONA & 39.6137 & 3.39227 & 20.20 & \pm 0.05 & 16.86 & \pm 0.05 & 20.44 & 15.37 & -0.24 & 1.49 \\
EIVI & 38.9512 & 1.40687 & 19.85 & \pm 0.05 & 16.46 & \pm 0.05 & 20.23 & 15.51 & -0.38 & 0.95 \\
FORM & 38.7053 & 1.42879 & 20.58 & \pm 0.04 & 16.67 & \pm 0.05 & 20.28 & 15.51 & 0.30 & 1.16 \\
JORD & 39.3149 & 2.99817 & 20.61 & \pm 0.05 & 16.38 & \pm 0.06 & 20.43 & 15.40 & 0.18 & 0.98 \\
MALL & 39.5526 & 2.62455 & 20.37 & \pm 0.05 & 16.57 & \pm 0.05 & 20.32 & 15.42 & 0.05 & 1.15 \\
MENC & 40.0006 & 3.83123 & 21.27 & \pm 0.05 & 16.00 & \pm 0.05 & 20.44 & 15.33 & 0.83 & 0.67 \\
SINE & 39.6459 & 3.01535 & 20.51 & \pm 0.05 & 16.41 & \pm 0.05 & 20.37 & 15.40 & 0.14 & 1.01 \\
TRAM & 39.8184 & 2.89161 & 20.05 & \pm 0.07 & 16,40 & \pm 0.08 & 20.31 & 15.41 & -0.26 & 0.99 \\
\hline
\end{tabular}

\section{Discusión}

La tendencia general de las velocidades absolutas en las Islas Baleares es hacia el nordeste, similar a la velocidad regional que presenta esta parte del Mediterráneo occidental. Las magnitudes del campo de velocidades absolutas oscilan entre 19.85$21.27 \mathrm{~mm} / \mathrm{año}$ en la componente Este y los 16.00-16.86 mm/año en la Norte. El promedio de velocidades está en $20.45 \mathrm{~mm} /$ año al Este y $16.45 \mathrm{~mm} /$ año al Norte. La red muestra una velocidad interna consistente en la mayoría de las estaciones, con pequeñas variaciones en dirección y magnitud en algunas de ellas. Los errores estimados son consistentes, con valores medios de aproximadamente $0.05 \mathrm{~mm} / \mathrm{año}$ en ambas componentes horizontales (Tabla 1).

La Fig. 3 muestra el campo de velocidades residuales en las Islas Baleares respecto al modelo teórico GEODVEL considerando la placa Euroasiática como referencia. Las componentes de velocidad residual en las estaciones son menores a $0.83 \mathrm{~mm} /$ año en dirección Este y $1.49 \mathrm{~mm} / \mathrm{año}$ en dirección Norte. Existe una correspondencia adecuada en prácticamente todas las estaciones, destacando las estaciones situadas en la zona central y occidental de Mallorca con direcciones 
prácticamente similares y pequeñas magnitudes. Los vectores de velocidad residual de las estaciones de MENC y BONA poseen una cierta correspondencia mientras que las velocidades de EIVI y FORM reflejan diferencias más acentuadas respecto a aquellas propias del modelo.

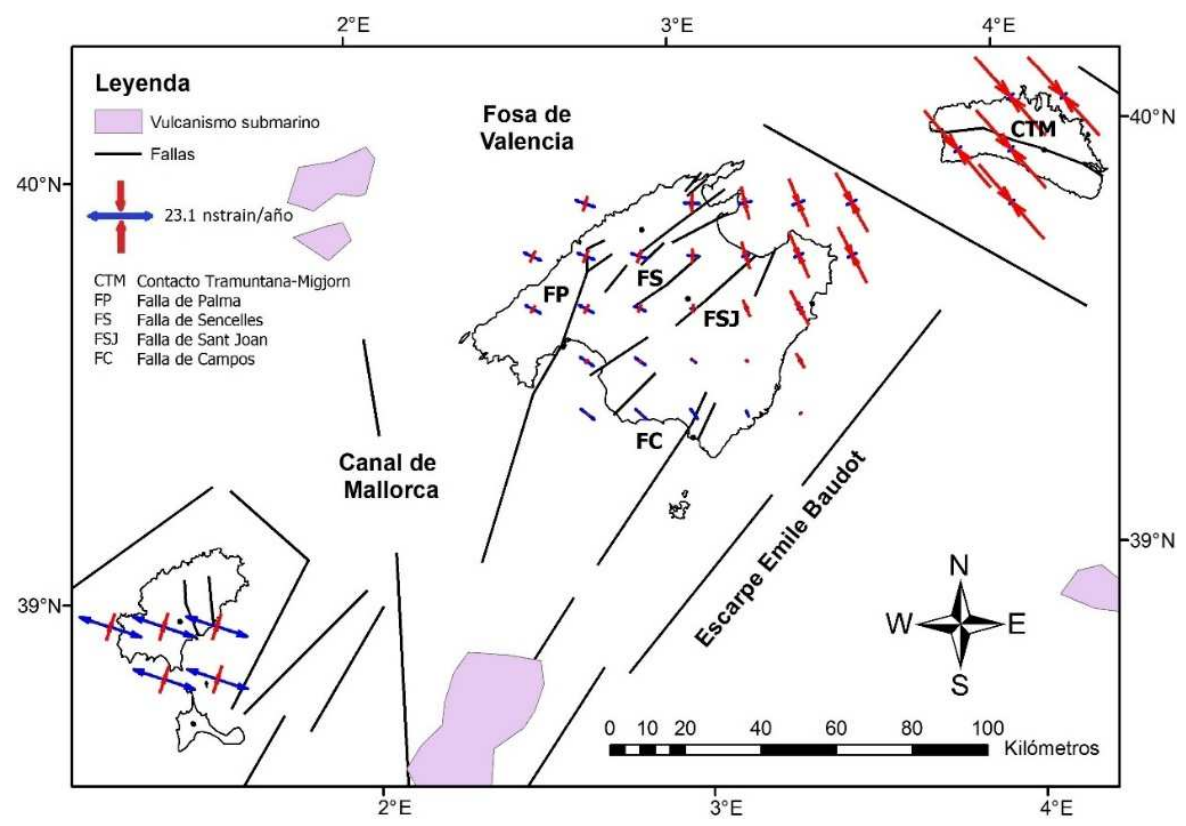

Fig. 4. Mapa de los ejes principales de los tensores de esfuerzo en nstrain/año. Los vectores en azul indican extensión y los rojos compresión en las direcciones correspondientes. Se indican las fallas principales presentes en el área.

Analizándose los esfuerzos calculados (Figs. 5 y 6) se tendrían los siguientes resultados: (1) acortamiento NW-SE en la Isla de Menorca y en la parte más oriental de Mallorca (BONA), (2) extensión E-W en la zona central de Mallorca (en las proximidades de las estaciones MALL y TRAM), (3) bajos índices de deformación en el sureste de Mallorca (en el entorno de la estación JORD), y (4) extensión WNW-ESE en Ibiza y Formentera. En general existe una variación desde la extensión WNW-ESE presente en las islas occidentales (Pitiusas) al acortamiento presente al nordeste (Menorca).

Los esfuerzos estimados están de acuerdo con los datos geológicos. En Menorca, el anticlinal NNE-SSW de Migjorn (MA Fig. 6) (Gelabert et al., 2005) que afecta a su vez al área septentrional de la isla, se puede relacionar con el acortamiento en dirección NW-SE inferido a partir de los datos geodésicos. En Mallorca, los anticlinales de Llucmajor y Santa Margalida, relacionados con las fallas normales NNE-SSW de Sa Pobla y Palma, son compatibles con la extensión E-W inferida a 
partir de las velocidades absolutas. Finalmente, la extensión WNW-ESE observada en las Islas Pitiusas puede ser atribuido a las fallas normales NE-SW y a las fallas NNW-SSE que controlan las estructuras de las islas de Ibiza, Formentera y las zonas submarinas anexas (Fig. 6).

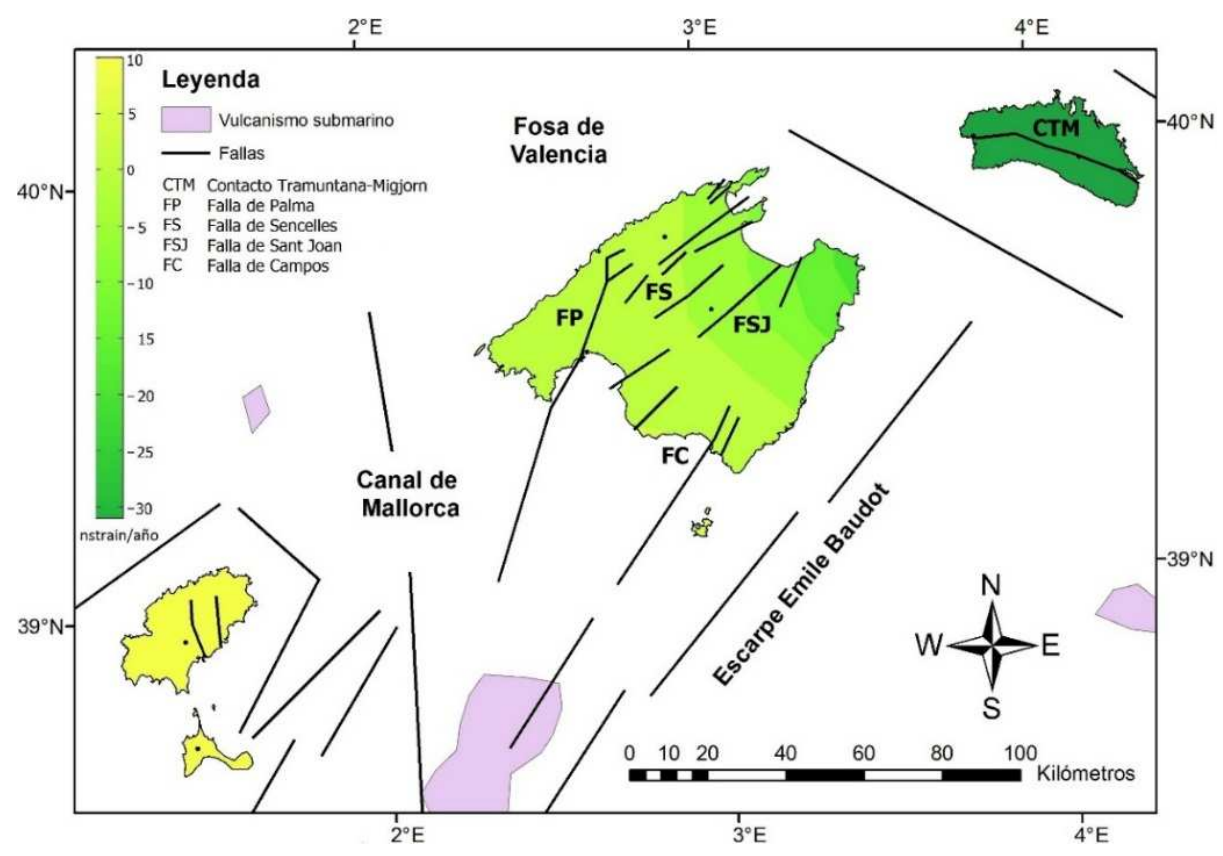

Fig. 5. Mapa de contorno con la representación de las áreas con predominancia de extensión (amarillo) y compresión (verde) en el Archipiélago Balear en nstrain/año. Se indican las fallas principales presentes en el área.

En base a los vectores de velocidad calculados es posible asignar las nueve estaciones GPS del archipiélago a seis bloques separados por las diversas fallas presentes: (1) La Isla de Menorca, (2) la Sierra de Tramuntana de Mallorca (estación TRAM), (3) las cuencas neógenas de la parte central de Mallorca, (4) la Sierra de Llevant de Mallorca (estación BONA), (5) la Isla de Ibiza y (6) la Isla de Formentera (Fig. 6). Estos seis bloques están controlados por regímenes generales de salto en dirección con acortamiento N-S y extensión E-W, estando separados por fallas normales sinistrorsas de dirección NNE-SSW a NE-SW y fallas normales dextras NW-SE.

La componente de velocidad hacia el Este en el bloque de la Isla de Menorca (bloque 1) es el de mayor magnitud, mientras que la componente hacia el Norte es la más pequeña. Estos valores son consistentes con un movimiento dextro en la falla NW-SE del Canal de Menorca (Fig. 6). La componente de velocidad hacia el Este 
de mayor magnitud que presenta el bloque central de la Isla de Mallorca (bloque 3) respecto al bloque de la Sierra de Tramuntana (bloque 2) es consistente con un movimiento sinistrorso de las fallas situadas en esa área (como por ejemplo la Falla de Sencelles). Los tres bloques definidos en la Isla de Mallorca tienen además una componente de velocidad más hacia el Este que los presentes en el bloque de Ibiza (bloque 5), lo que se atribuye a un movimiento dextro de las fallas NW-SE del Canal de Mallorca. Los movimientos extensionales en las proximidades de las fallas $\mathrm{N}-\mathrm{S}$, o el movimiento sinistrorso de las fallas NE-SW localizadas entre las islas de Ibiza y Formentera, es consistente con un vector de velocidad hacia el Este más grande del bloque de Formentera respecto al de Ibiza. Por último, La mayor componente hacia el Norte del bloque correspondiente a las Sierras del Llevant respecto al bloque de la zona central de Mallorca puede estar relacionado con el movimiento sinistrorso de la falla NNE-SSW en las proximidades de las Sierras del Llevant.

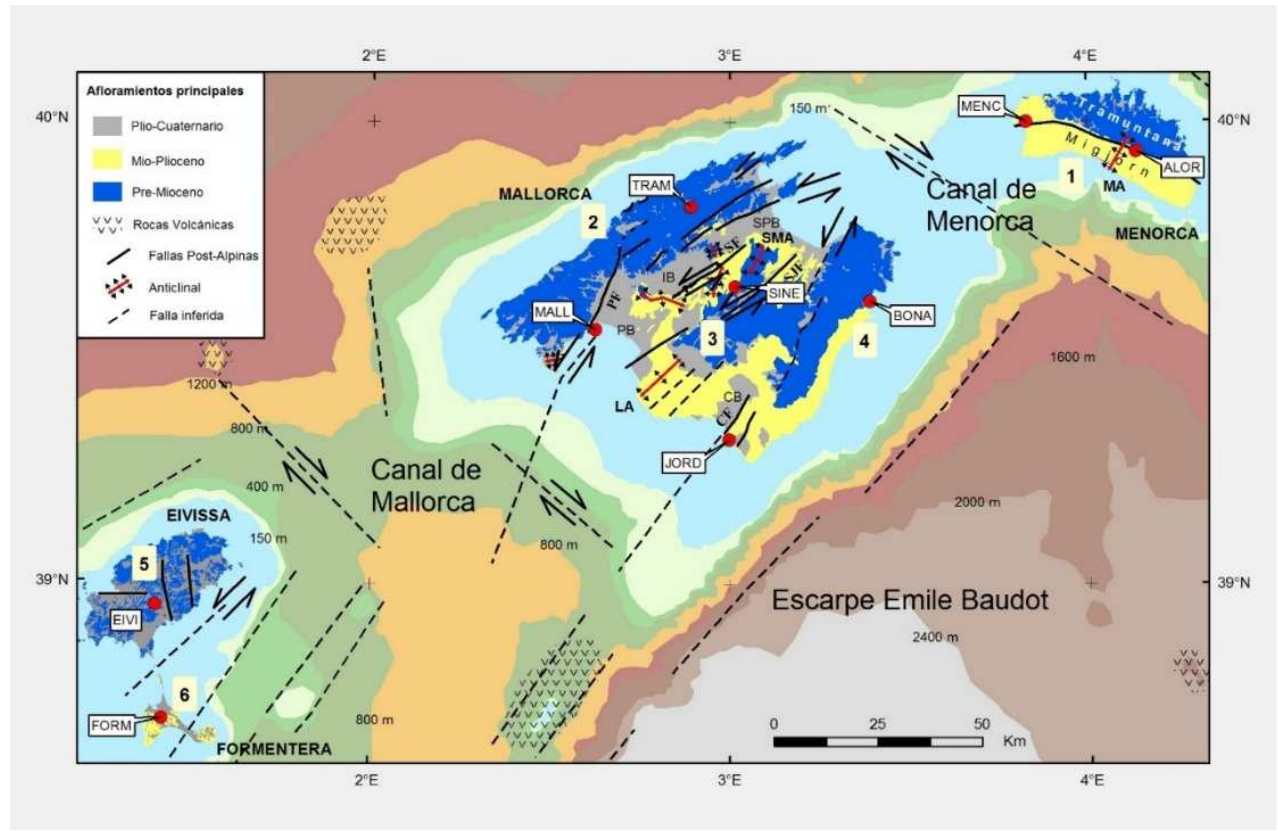

Fig. 6. Interpretación geodinámica de las velocidades obtenidas. Quedan reflejadas las fallas y las cuencas neógenas más importantes (PF: Falla de Palma; SF: Falla de Sencelles; SJF: Falla de San Joan; CF: Falla de Campos; LA: Anticlinal de Llucmajor; SMA: Anticlinal de Santa Margalida; PB: Cuenca de Palma; SPB: Cuenca de Sa Pobla; CB: Cuenca de Campos) 


\section{Conclusiones}

Esta primera cuantificación de la deformación cortical de las Islas Baleares integrada con la información geológica es de gran utilidad para los estudios geodinámicos regionales en la zona más occidental del Mediterráneo. Los campos de velocidades y esfuerzos son consistentes con la distribución de las estructuras geológicas presentes. Existen sin embargo pequeñas discrepancias que se pueden atribuir al corto intervalo de tiempo de los datos geodésicos disponibles en el área, donde se esperan velocidades residuales menores a $1 \mathrm{~mm} / \mathrm{año}$ (generalmente menores a $0.5 \mathrm{~mm} /$ año). Los trabajos futuros en esa dirección, integrando nuevos datos geodésicos, estudios geológicos, geofísicos y la información sísmica proveniente de terremotos acaecidos en las proximidades, ayudarán a una mejor compresión de la geodinámica presente en esta zona.

\section{Agradecimientos}

Esta investigación ha sido parcialmente financiada por el Ministerio de Ciencia e Innovación a través de los proyectos AYA2010-15501, CGL2011-30153-C02-02 y CSD2006-0041 (Fondo de Desarrollo Regional Europeo ERDF). Los autores quieren agradecer al Serveis d'Informació Territorial de les Illes Balears (SITIBSA) por facilitar los ficheros de observación de la red XGAIB. Algunas figuras incluidas han sido generadas utilizando el programa Generic Map Tool (GMT) (Wessel y Smith, 1998) y el software MGDS Global Multi-Resolution Topography.

\section{Referencias Bibliográficas}

ACOSTA, J., MUÑOZ, A., HERRANZ, P., PALOMO, C., BALlESTEROS, M., VAQUERO, M. \& UCHUPI, E., (2001). Geodynamics of the Emile Baudot escarpment and the Balearic promontory, western Mediterranean. Marine and Petroleum Geology 18, 349-369.

ACOSTA, J., CANALS, M., LÓPEZ-MARTÍNEZ, J., MUÑOZ, A., HERRANZ, P., URGELES, R., PALOMO, C. \& CASAMOR, J. L., (2002). The Balearic Promontory geomorphology (western Mediterranean): morphostructure and active processes. Geomorphology 49, 177-204.

ACOSTA, J., ANCOCHEA E., CANALS M., HUERTAS, M.J. \& UCHUPI, E., (2004). Early Pleistocene volcanism in the Emile Baudot Seamount, Balearic Promontory (western Mediterranean Sea). Marine Geology 207, 247-257.

ARGUS, D.F. \& GORDON, R.C., (1991). No-net-rotation model of current plate velocities incorporating plate rotation model NUVEL-1. Geophysical Research Letters 18, 2039-2042.

ARGUS, D.F., GORDON, R.C., HEFLIN, M.B., MA, C., EANES, R.J., WILLIS, P., PELTIER, W.R. \& OWEN, S.E., (2010). The angular velocities of the plates 
and the velocity of Earth's Centre form space geodesy. Geophysical Journal International 180, 913-960.

BERTIGER, W., DESAI, S., DORSEY, HAINES, B., HARVEY, N., MOORE, A., OWEN, S. \& WEISS, J.P., (2010). Single receiver phase ambiguity resolution with GPS data. Journal of Geodesy 84, 327-337.

BILITZA, D. (2001). International Reference Ionosphere 2000. Radio Science, 36(2): 261-275.

BOEHM, J., WERL, B., \& SCHUH, H. (2006). Troposphere mapping functions for GPS and very long baseline interferometry from European Centre for Medium Range Weather Forecasts operational analysis data. Journal of Geophysical Research, 111

BRAUNMILLER, J., KRADOLFER, U., BAER, M. \& GIARDINI, D., (2000). Regional moment-tensor inversion in the European-Mediterranean Area. Orfeus Electronic Newsletter, 2 (1), 5

CALAIS, E., DEMETS, C. \& NOCQUET, J.M., (2003). Evidence for a post-3.16 Ma. change in Nubia-Eurasia-North America plate motions? Earth and Planetary Science Letters 160, 651-665.

DEWEY, J.F., HELMAN, M.L., TURCO, E., HUTTON, D.H.W. \& KNOTT, S.D., (1989). Kinematics of the western Mediterranean. In Coward M.P.D. Park R.G. (Eds.), Alpine Tectonics: Geological Society. London Special Publication 45, 265-283.

DEMETS, C., GORDON, R., ARGUS, D. \& STEIN, S., (1994). Effect of recent revisions to the geomagnetic reversal time scale on estimates of current plate motions. Geophysical Research Letters 21, 2191-2194.

FADIL, A., VERNANT, P., MCCLUSKY, S., REILINGER, R., GOMEZ, F., BEN SARI, D., MOURABIT, T., FEIGL, K.L. \& BARAZANGI, M., (2006). Active tectonics of the western Mediterranean: GPS evidence for roll back of a delaminated subcontinental lithospheric slab beneath the Rif Mountains. Morocco. Geology 34, 529-532.

FERNANDES, R., MIRANDA, J., NEIJNINGER, B., BOS, M., NOOMEN, R., BASTOS, L., AMBROSIUS, B. \& RIVA, R., (2007). Surface velocity field of the Ibero Maghrebian segment of the Eurasia Nubia plate boundary. Geophysical Journal International 169, 315-324.

GELABERT, B., FORNÓS, J.J., PARDO, J.B., ROSSELLÓ, V.M. \& SEGURA, F., (2005). Structurally controlled drainage basin development in the south of Menorca (western Mediterranean, Spain). Geomorphology 65, 139-155.

GIMÉNEZ, J., (2003). Nuevos datos sobre la actividad post-Neógena en la Isla de Mallorca. Geogaceta 33, 79-82.

GIMÉNEZ, J. \& GELABERT, B., (2002). Análisis de la actividad tectónica reciente en la isla de Mallorca. III Asamblea Hispano Portuguesa de Geodesia y Geofisica. 
GRAFAREND, E.W. \& VOOSOGHI, B., (2003). Intrinsic deformation analysis of the Earth's surface based on displacement fields derived from space geodetic measurements. Case studies: Present-day deformation patterns of Europe and of the Mediterranean area (ITRF data sets). Journal of Geodesy 77, 303-326.

HREINSDÓTTIR, S., FREYMUELLER, J.T., BÜRGMANN, R. \& MITCHELL, J., (2006). Coseismic deformation of the 2002 Denali fault earthquake: Insights from GPS measurements. Journal of Geophysical Research 111.

KOCH, K.R., (1999). Parameter Estimation and Hypothesis Testing in Linear Models. Springer-Verlag. Berlin.

KOUBA, J., (2005). A possible detection of the 26 December 2004 great SumatraAndaman Islands earthquake with solution products of the International GNSS Service. Studia Geophysica et Geodaetica 49, 463-483.

KOULALI, A., OUAZAR, D., TAHAYT, A., RING, R.W., VERNANT, P., REILINGER, R.E., MCCLUSKY, S., MOURABIT, T., DAVILA, J.M. \& AMRAOUI, N., (2011). New GPS constraints on active deformation along the Africa-Iberia late boundary. Earth and Planetary Science Letters 308, 211-217.

LARSON, K.M., LOWRY, A.R., KOSTOGLODOV, V., HUTTON, W., SÁNCHEZ O., HUDNUT, K. \& SUÁREZ, G., (2004). Crustal deformation measurements in Guerrero, Mexico. Journal of Geophysical Research 109, 119.

LYARD, F., LEFEVRE, F., LETELLIER, T. \& FRANCIS, O., (2006). Modelling the global ocean tides: Modern insights from FES2004. Ocean Dynamics 55, 394-415.

MAILLARD, A. \& MAUFFRET, A., (2013). Structure and present-day compression in the offshore area between Alicante and Ibiza island (eastern Iberian margin). Tectonophysics 591, 116-130.

MCCLUSKY, S., REILINGER, R., MAHMOUD, S., SARI, D.B. \& TEALEB, A., (2003). GPS constraints on Africa (Nubia) and Arabia plate motions. Geophysical Journal International 155 (1), 126-138.

NOCQUET, J.M. \& CALAIS, E., (2003). Crustal velocity field of Western Europe from permanent GPS array solutions, 1996-2001. Geophysical Journal International 154, 72-88.

PALANO, M., CANNAVO, F., FERRANTI, L., MATTIA, M. \& MAZZELLA, E., (2011). Strain and stress fields in the southern Apennines (Italy) constrained by geodetic, seismological and borehole data. Geophysical Journal International 187, 1270-1282.

PENNA, N.T. \& STEWART, M.P., (2003). Aliased tidal signatures in continuous GPS height time series. Geophysical Research Letters 30, 2184.

PÉREZ, J.A.S., MONICO, J.F.G. \& CHAVES, J.C., (2003). Velocity field estimation using GPS Precise Point Positioning: The South American Plate Case. Journal of Global Positioning Systems 2, 90-99. 
PEREZ-PEÑA, A., MARTÍN-DÁVILA, J., GÁRATE, J., BERROCOSO \& M., BUFORN, E., (2010). Velocity field and tectonic strain in southern Spain and surrounding areas derived from GPS episodic measurements. Journal of Geodynamics 49, 232-240.

PIETRANTONIO, G. \& RIGUZZI, F., (2004). Three-dimensional strain tensor estimation by GPS observations: Methodological aspects and geophysical applications. Journal of Geodynamics 38, 1-18.

ROCA, E., (1996). La evolución geodinámica de la Cuenca Catalano-Balear y áreas adyacentes desde el Mesozoico hasta la actualidad. Acta Geologica Hispánica $29,3-25$.

SERPELLONI, E., VASNNUCCI, G., PONDRELLI, S., ARGNANI, A., CASULA, G., ANZIDEI, M., BALDI, P. \& GASPERINI, P., (2007). Kinematics of the western Africa-Eurasia plate boundary from focal mechanisms and GPS data. Geophysical Journal International 169, 1180-1200.

SÀBAT, F., GELABERT, B., RODRÍGUEZ-PEREA, A. \& GIMÉNEZ, J., (2011). Geological structure and evolution of Majorca: Implications for the origin of the western Mediterranean. Tectonophysics 510, 217-238.

SILVA, P.G., GONZÁLEZ-HERNÁNDEZ, F.M., GOY, J.L. \& ZAZO, C., (1997). Origen y desmantelamiento del Antiforme Plio-Cuaternario de Marratxí (Mallorca, España). Geogaceta 22, 143-147.

SILVA, P.G., GONZÁLEZ-HERNÁNDEZ, F.M., GOY, J.L., ZAZO, C. \& MÖRNER, N.A., (1999). Quaternary reverse surface faulting in Mallorca Island (Baleares, Spain). Geogaceta 26, 99-102.

SILVA. P.G., CARRASCO, P., GONZÁlEZ HERNÁNDEZ, F.M., GOY, J.L., ZAZO, C., LUQUE, L., SANTOS,G., DELGADO, M. \& POZA, L.J., (2000). Prospección geofísica de la Falla de Sencelles (Mallorca, España): Una metodología preliminar para la realización de trincheras de falla. Geotemas 1(4), 359-363.

SILVA, P.G., GONZÁLEZ-HERNÁNDEZ, F.M., GOY, J.L., ZAZO, C. \& CARRASCO, P., (2001). Paleo and historical seismicity in Mallorca (Baleares, Spain): A preliminary approach. Acta Geológica Hispánica 36 (3-4), 245-266.

SILVA, P.G., GONZÁLEZ HERNÁNDEZ F.M., GIMÉNEZ, J., SANTOS, G., CARRASCO, P., DE LUQUE, L., DELGADO, M., GOY, J.L. \& ZAZO, C., (2004). Fault-trenching analysis of the Sencelles fault (Mallorca island, Spain). $32^{\text {nd }}$ Internacional Geological Congreso.

SILVA, P.G., MATEOS, R., GOY, J.L., ZAZO, C., GONZÁLEZ-HERNÁNDEZ, F.M., GIMÉNEZ, J., FORNÓS, J., HILLAIRE-MARCEL, CL., CABERO, A., BASSAM, G. \& BARDA, T., (2005). Mallorca island: Geomorphological evolution and neotectonics. Field Trip Guides of the 6th International Conference on Geomorphology IAG-SEG Zaragoza. Vol. II, 433-472. 
SMITH, K.D., VON SEGGERN, D., BLEWITT, G., PRESTON, L., ANDERSON, J.G., WERNICKE, B.P. \& DAVIS, J.L., (2004). Evidence for deep magma injection beneath lake Tahoe, Nevada-California. Science 305, 1277-1280.

STICH, D., SERPELLONI, E., MANCILLA, F. \& MORALES, J., (2006). Kinematics of the Iberia-Maghreb plate contact from seismic moment tensors and GPS observations. Tectonophysics 426, 295-317.

TAHAYT, A., MOURABIT, T., RIGO, A., FEIGL, K.L., FADIL, A., MCCLUSKY, S., REILINGER, R., SERROUKH, M., OUAZZANITOUHAMI, A., BEN-SARI, D. \& VERNANT, P., (2008). Mouvements actuels des blocs tectoniques dans l'arc Bético-Rifain à partir des mesures GPS entre 1999 et 2005. Comptes Rendus Geoscience 340, 400-413.

TEFERLE, F.N., ORLIAC, E.J. \& BINGLEY, R.M., (2007). An assessment of Bernese GPS software precise point positioning using IGS final products for global site velocities. GPS solutions 11, 205-213.

TEZA, G., PESCI, A. \& GALGARO, A., (2008). Grid_strain and grid_strain3: Software packages for strain field computation in 2D and 3D environments. Computers \& Geosciences 34, 1142-1153.

VERNANT, P., FADIL, A., MOURABIT, T., OUAZAR, D., KOULALI, A., DAVILA, J.M., GARATE, J., MCCLUSKY, S. \& REILINGER, R., (2010). Geodetic constraints on active tectonics of the western Mediterranean: Implications for the kinematics and dynamics of the Nubia-Eurasia plate boundary zone. Journal of Geodynamics 49, 123-129.

VOOSOGHI, B., (1976). Intrinsic Deformation Analysis of the Earth Surface Based on 3-Dimensional Displacement Fields Derived from Space Geodetic Measurements. PhD Thesis. University of Stuttgart.

WAHR, J. M. (1985). Deformation induced by Polar Motion. Journal of Geophysical Research, 90(B11):9363.9368

WESSEL, P. \& SMITH, W.H.F., (1998). New improved version of the Generic Mapping Tools Released. EOS Trans. AGU 79, 579.

WILLIAMS, S.D.P., (2008). CATS: GPS coordinate time series analysis software. GPS solutions 12, 147-153.

ZUMBERGE, J.F., HEFLIN, M.B., JEFFERSON, D.C., WATKINS, M.M. \& WEBB, F.H., (1997). Precise Point Positioning for the efficient and robust analysis of GPS data from large networks. Journal of Geophysical Research $102,5005-5017$. 